\title{
ASSESSMENT OF THE RELATIONSHIP BE- TWEEN TEMPOROMANDIBULAR JOINT SOUNDS AND BRUXISM
}

\author{
Dimitar Kirov $^{1}$, Lilia Doichinova ${ }^{2}$, Elitsa Deliverska ${ }^{3}$, Janet Kirilova ${ }^{4}$ \\ 1) Department of Prosthetic Dental Medicine, Faculty of Dental Medicine, Medi- \\ cal University, Sofia, Bulgaria \\ 2) Department of Pediatric Dental Medicine, Faculty of Dental Medicine, Medi- \\ cal University, Sofia, Bulgaria \\ 3) Department of Oral and Maxillofacial Surgery Faculty of Dental Medicine, \\ Medical University, Sofia, Bulgaria \\ 4) Department of Conservative Dentistry, Faculty of Dental Medicine, Medical \\ University, Sofia, Bulgaria.
}

\section{SUMMARY:}

Aim of the study: The study aims to assess the possible relationship between bruxism and TMJ sounds.

Material and methods: The relationship between temporomandibular joint (TMJ) sounds and bruxism was evaluated in a sample composed of 62 females and 39 males varied in age from 21 to 56 years with a mean age of $40.0 \pm 1.07$. The participants were informed about the purpose of this study, and they gave their consent. The main methods we used in registering TMJ sound were palpation and auscultation. A self-administered Bruxism Assessment Questionnaire assessed bruxism.

Results: The prevalence of bruxism in study group was $60.39 \%(n=61)$ - sleep bruxism $(\mathrm{SB})$ and awake bruxism $(\mathrm{AB})$ were $38.2 \%$ and $23.4 \%$, respectively. A total of $53.47 \%(n=54)$ of the sample exhibited some type of clicking joint sound. Chi-square test for independence (with Yates Continuity Correction) indicated significant association between bruxism and TMJ sounds, $\chi 2(1, n=101)=$ 4.183, $p=.046, p h i=.28$.

Conclusions: Biomechanical factors seem to play a significant role in TMJ disk displacement development. Based on the research, it should be concluded that bruxism may be associated with joint pathologies, such as disc displacement and joint noises.

Keywords: bruxism, disc displacement, teeth grinding, temporomandibular disorders, TMJ sounds,

\section{INTRODUCTION}

Typically temporomandibular disorder (TMD) is defined as a "group of disorders involving the masticatory muscles, the temporomandibular joint (TMJ), and the associated structures" [1]. They are primarily characterized by joint and muscular pain, noise in the temporomandibular joints (TMJ), and limited mandibular movements. Although the typical temporomandibular joint is described as silent, sounds can be heard in individuals who may or may not present pain symptoms [2]. Clinically, TMJ noise is related to the internal derangement of TMJ [3].

Temporomandibular joint (TMJ) sounds are the main symptoms of temporomandibular disorders. The TMJ internal derangement is an abnormal positional relationship between the disc and the condyle, articular eminence, and articular fossa [4]. Abnormal positioning may cause TMJ sounds and pain and may limit jaw function. The movement of the disc may result in a clicking, snapping, and popping sound known as an opening and closing click.

Articular sounds of the clicking type as during mouth opening and closing are characteristic clinical signs of disc displacement with reduction (DDWR) [5]. TMJ clicking corresponds from 18 to $26.2 \%$ of clinical symptoms of TMD and is one of the most common complaints of patients. According to a systematic review, $14.0 \%$ of the children or adolescents had clinical TMJ sounds[6], while a current investigation detected an even higher percentage up to $31.9 \%$ for German adolescents [7].

The American Academy of orofacial pain (AAOP) defined bruxism as "diurnal or nocturnal parafunctional activity including clenching, bracing, gnashing, and grinding of the teeth." The published data reveal that the prevalence of bruxism in students in Brazil was $31.6 \%$ in this population [8]. The most associated factors with bruxism were stress, muscle pain, TMJ pain, and TMJ noise.

The most common symptom of joint disfunction is TMJ clicking (30.7\%), followed by TMD pain (16.3\%) and TMJ crepitus (10.3\%) [9]. DDWR can occur in 33\% of asymptomatic individuals [10]. Among the intra-articular disorders of the TMJ, disc displacement with reduction (DDWR) corresponds to $41 \%$ of TMD clinical diagnoses [11]. TMJ clicking corresponds to $26.2 \%$ of clinical signs of TMD and is one of the most common complaints of patients [12].

Based on the evidence, the relationship between bruxism and TMD is still controversial in the literature due 
to the complexity of the etiology and diagnostic of both disorders [13-15].

All in all, the risk factors for TMJ sounds are still not fully understood, and biomechanical and anatomical causes seem to affect changes in the TMJ.

This study aimed to assess the possible relationship between bruxism and TMJ sounds.

\section{MATERIAL AND METHODS}

One hundred and thirty (130) participants with temporomandibular joint (TMJ) sounds were preliminarily selected for this clinical study. The association between joint sounds and bruxism we evaluated in a sample composed of 71 females and 59 males ranging in age from 21 to 56 years with a mean age of $36.2 \pm 1.0$. Before the clinical trial, we notified the patients about the purpose of this study, and all participants gave their consent. In addition, all participants completed self-administered questionnaires consisting of questions about bruxism. According to RDC/TMD Axis IIa diagnostic criteria, the patients underwent routine clinical examination. It was performed only by one expert investigator to minimize error.

\section{TMJ sounds diagnosis}

Trained dentists examined temporomandibular joint sounds according to the Diagnostic Criteria for Temporomandibular Disorders (DC/TMD) [3]. The DC/TMD, which is a standardized tool to diagnose TMD, presents the following diagnostic criteria for DDWR and presence for at least one of the following symptoms:

" TMJ noise(s) present with jaw movement or function in the last month;

"Clicking, popping, or snapping noise detected with palpation during at least one of three repetitions of opening or closing movement(s);

"Clicking, popping, or snapping noise detected with palpation during at least one of three lateral or protrusive movement(s).

The main symptom of disc displacement with reduction is a click sound during jaw movements. It must be reciprocal (audible during both jaw opening and jaw-closing activities) and not fixed (loud during the jaw opening and jaw-closing movements).

\section{Bruxism Diagnosis}

The study exclusively used self-reports in bruxism diagnosis for identifying possible bruxers. Sleep bruxism (SB) was evaluated by a questionnaire recommended by the American Academy of Sleep Medicine [16] as follow:

1. Are you aware of grinding your teeth frequently during sleep, or has anyone heard you? (yes/no)

2. Are you consider that your dentition is worn down more than normal? (yes/no)

3. Are you familiar with any of the following signs and symptoms upon awakening? (yes/no):

- Sensation of fatigue, tightness, or soreness of your jaw upon awakening?

- Feeling that your teeth are clenched or that your mouth is sore upon awakening?

- Aching of your temples upon awakening?

- Difficulty in opening your mouth wide upon awakening?

- Tension in your jaw joint upon awakening and feeling as if you have to move your lower jaw to release it?

- Hearing or feeling a "click" in your jaw joint upon awakening that disappears afterward?

4. Do you grind your teeth or clench your jaws during wakefulness? (yes/no)

The respondents were determined to suffer from possible SB if their answer to question 1 or 2 was "yes" or at least one "yes" answer to a symptom listed in question 3. The "yes " response to Question 4 indicated that the participant had possible AB.

\section{Statistical analysis}

Statistical analysis was performed using the IBM SPSS Statistics software package (IBM SPSS Statistics for Windows, Version 20.0. Armonk, NY: IBM Corp). Descriptive methods of categorical and quantitative variables (mean, median, standard deviation, minimum and maximum) were used. The relationship between bruxism and occlusal factors was evaluated with the chi-square test.

\section{RESULTS}

There were 54 participants $(53.47 \%)$ diagnosed with TMJ sounds. The prevalence of bruxism was $60.39 \%(\mathrm{n}=$ $61)-\mathrm{SB}$ and $\mathrm{AB}$ were $37.2 \%$ and $23.19 \%$, respectively. No significant difference was observed between females and males for both TMJ sounds $(\mathrm{p}=0.852)$ and bruxism $(0.448)$ (Table 1).

Table 1. Descriptive statistics of bruxism and TMJ sounds by gender among the study group and crosstabulation

\begin{tabular}{lcccccc}
\hline & \multicolumn{3}{c}{ Gender } & \multicolumn{2}{c}{$\chi^{2}$ test } \\
\hline \multirow{4}{*}{ TMJ sounds } & & Male & Female & Total & Value & df \\
& Yes & 19 & 35 & 54 & & \\
& No & 20 & 27 & 47 & & \\
& Total & 39 & 62 & 101 & 0.576 & 1 \\
\hline \multirow{5}{*}{ Bruxism } & & Male & Female & Total & Value & df \\
& Yes & 24 & 37 & 61 & & \\
& No & 15 & 25 & 40 & & \\
& Total & 39 & 62 & 101 & 0.034 & 1 \\
\hline
\end{tabular}


Correlation analysis was used to identify the correlation between the TMJ sounds and bruxism. Chi-square test for independence (with Yates Continuity Correction) indicated significant association between bruxism and TMJ sounds, $\chi 2(1, n=101)=4.183, p=.046$, phi $=$
.28. We found that the size of the effect of the above indicators is close to medium based on benchmarks suggested by Cohen (.10 for small effect, .30 for medium effect, and .50 for large effect). Results of the analysis are reported in Table 2.

Table 2.Association and effect size between bruxism and TMJ sounds

\begin{tabular}{lcccc}
\hline & & \multicolumn{3}{c}{ Asymp. Sig. } \\
& Value & df & (2-sided) & Phi \\
\cline { 2 - 5 } Pearson Chi-Square & 4.827 & 1 & .028 & \\
Continuity Correction & 3.972 & 1 & .046 & 0.028 \\
\hline
\end{tabular}

The results of this study demonstrate a positive relationship between bruxism and TMJ sounds.

Fig. 1. Distribution of bruxism and TMJ sounds among the study population.

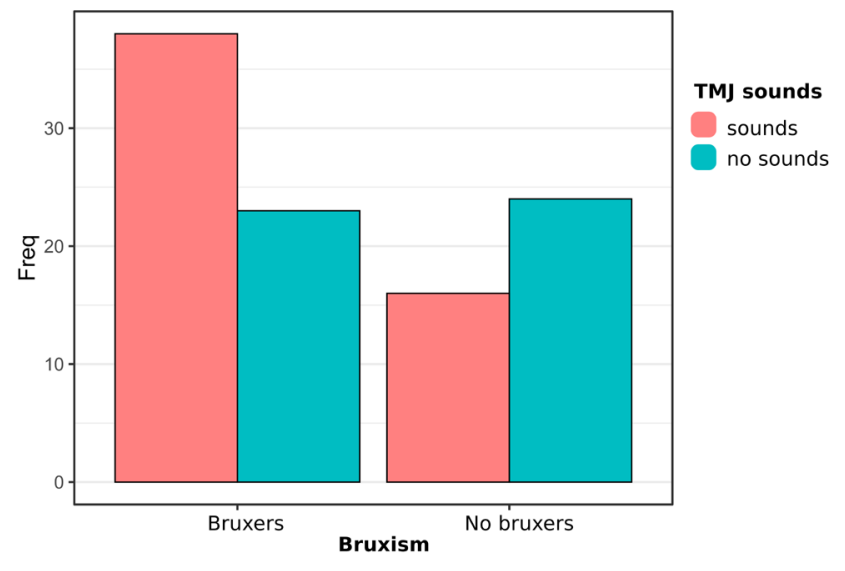

A positive correlation sign indicates that the increase in the size of factor bruxism enhances the possibility of TMJ sounds.

\section{DISCUSSION}

This study tries to assess the possible relationship between bruxism and internal derangement of TMJ caused by mechanical overloading. Many clinical studies have investigated this correlation in recent years, producing a range of contradictory results and conclusions. Based on the evidence, the relationship between bruxism and TMD is still controversial in the literature. That may be due to the complexity of the etiology and diagnostic of both disorders [13].

Most studies explained a TMJ sound by the movement of the articular disc onto the condyle during jaw movements. This pathological movement is caused by displacing the articular disc with reduction (DDWR) [10]. Disc disorders (DD) is a condition that implicates no discomfort for the patient, yet it can induce pain or limitations in jaw movements during the mastication process $[17,18]$. Our data agree with the research carried out by Marpaung $\mathrm{C}$ et al., which showed that biomechanical factors play a significant role in TMJ disk displacement development [19].
Earlier studies [20,13] hypothesized that such symptoms might be caused by anatomical and functional disturbances in the TMJ. However, several studies suggest that clenching and grinding, especially during the daytime, are positively correlated with disc displacement and TMJ sounds, consistent with our results.

The shear stresses estimated in research for bruxism, but especially those obtained in sustained clenching can cause damage in the articular disc, leading to TMDs [21].

Although the studies showed a high risk of bias, the qualitative analysis of individual studies showed that children with bruxism have a greater chance of developing TMD [14] and prospectively displacing TMJ disc.

Reports show that bruxism was more often associated with TMJ symptoms, increasing the risk of TMJ pain, noise, and limited mouth opening. Based on research [22], we concluded that excessive load affecting temporomandibular joints caused by chewing and occlusal forces generated during parafunction and in people with defined longterm bruxism has crucial importance on biomechanical disc properties and hence the course of temporomandibular joint conditions. The results of previous reports significantly associated crepitus and temporomandibular joint sounds with oral behaviors [9]. An identical conclusion was reached by other studies showing that stress, muscle pain, temporomandibular joint (TMJ) pain, and TMJ noise were significantly associated with bruxism [8].

The analysis of short and long-term studies showed that parafunctional habits, such as bruxism, have been associated with hyperactivity of the masticatory muscles. The resultant loading to the temporomandibular joint (TMJ) is subject to changes in cartilage and disc [23]. The study explains the effect of clenching direction on the stress distribution in the TMJ in parafunction might result in increased stresses on the TMJ disc and the condylar and temporal articular surfaces. A previous study established that the higher stresses are concentrated in the lateral region during prolonged clenching. That could imply that TMJ disorders are related to damage or wear in the disc and the condylar cartilage occur when dysfunctional displacements are present [24].

According to other authors, the interaction between sleep and awake bruxism may increase joint sounds and TMD [25]. Among harmful oral habits, bruxism has been 
considered a vital maintenance factor of TMD. It appears that TMD pain and TMJ clicking are significantly associated with oral behaviors [26].

\section{CONCLUSION}

Within the limitations of this study, we concluded that oral parafunction, especially bruxism, was associated with joint pathology as disc displacement and joint noises. TMJ disc displacement and TMJ sounds are commonly asymptomatic conditions, and no treatment is usually re- quired since the structures in this region may change and adapt. Treatment should be done when the noise is accompanied by pain. Bruxism generally appears to be a significant factor in contributing to TMJ disorders. A clinical investigation should be performed to confirm and generalize these findings.

\section{Consent for publication}

Written informed consents for publication of any associated data were obtained from all patients.

\section{REFERENCES:}

1. deLeeuw R, Klasser GD. Orofacial Pain: Guidelines for Assessment, Diagnosis, and Management (AAOP The American Academy of Orofacial Pain), 6th Edition. Quintessence Pub. Co. April 10, 2018. 336 p. [Internet]

2. Kaymak D, Karakis D, Dogan A. Evolutionary Spectral Analysis of Temporomandibular Joint Sounds Before and After Anterior Repositioning Splint Therapy in Patients with Internal Derangement. Int J Prosthodont. 2019 Nov-Dec;32(6):475-481. [PubMed]

3. Schiffman E, Ohrbach R, Truelove E, Look J, Anderson G, Goulet JP, et al. Diagnostic Criteria for Temporomandibular Disorders (DC/TMD) for clinical and research applications: recommendations of the International RDC/TMD Consortium Network and Orofacial Pain Special Interest Group. J Oral Facial Pain Headache. 2014; 28(1):6-27. [PubMed]

4. Ohrbach R, Dworkin SF. The Evolution of TMD Diagnosis: Past, Present, Future. J Dent Res. 2016 Sep;95(10):1093-101. [PubMed]

5. Xie C, Lin M, Yang H, Ren A. Prevalence of temporomandibular disorders and its clinical signs in Chinese students, 1979-2017: A systematic review and meta-analysis. Oral Dis. 2019 Oct;25(7):1697-1706. [PubMed]

6. Da Silva CG, Pachêco-Pereira C, Porporatti AL, Savi MG, Peres MA, Flores-Mir C, et al. prevalence of clinical signs of intra-articular temporomandibular disorders in children and adolescents: A systematic review and meta-analysis. J Am Dent Assoc. 2016; 147:10-18. [PubMed]

7. Rauch A, Schierz O, Körner A,
Kiess W, Hirsch C. Prevalence of anamnestic symptoms and clinical signs of temporomandibular disorders in adolescents-Results of the epidemiologic LIFE Child Study. J. Oral Rehabil. 2019;47:425-431. [PubMed]

8. Soares LG, Costa IR, Brum Júnior JDS, Cerqueira WSB, Oliveira ES, Douglas de Oliveira DW, et al. Prevalence of bruxism in undergraduate students. Cranio. 2017 Sep;35(5): 298-303. [PubMed]

9. Iodice G, Cimino R, Vollaro S, Lobbezoo F, Michelotti A. Prevalence of temporomandibular disorder pain, jaw noises and oral behaviours in an adult Italian population sample. $J$ Oral Rehabil. 2019 Aug;46(8):691698. [PubMed]

10.Poluha RL, Canales GT, Costa YM, Grossmann E, Bonjardim LR, Conti PCR. Temporomandibular joint disc displacement with reduction: a review of mechanisms and clinical presentation. J Appl Oral Sci. 2019; 27:e20180433. [PubMed]

11. Talaat WM, Adel OI, Al Bayatti S. Prevalence of temporomandibular disorders discovered incidentally during routine dental examination using the Research Diagnostic Criteria for Temporomandibular Disorders. Oral Surg Oral Med Oral Pathol Oral Radiol. 2017;125(3):250-259. [PubMed]

12.Jussila $\mathrm{P}$, Kiviahde $\mathrm{H}$, Näpänkangas R, Päkkilä J, Pesonen P, Sipilä K, et al. prevalence of temporomandibular disorders in the Northern Finland Birth Cohort 1966. J Oral Facial Pain Headache. 2017; 31(2):159-164. [PubMed]

13.Jimenez-Silva A, Peea-Duran C, Tobar-Reyes J, Frugone-Zambra R. Sleep and awake bruxism in adults and its relationship with temporomandibular disorders: A systematic review from 2003 to 2014. Acta Odontol Scand. 2017 Jan;75(1):36-58. [PubMed]

14. de Oliveira Reis L, Ribeiro RA, Martins CC, Devito KL. Association between bruxism and temporomandibular disorders in children: A systematic review and meta-analysis. Int J Paediatr Dent. 2019 Sep;29(5): 585595. [PubMed]

15. Atsu SS, Guner S, Palulu N, Bulut AC, Kurkcuoglu I. Oral parafunctions, personality traits, anxiety and their association with signs and symptoms of temporomandibular disorders in the adolescents. Afr Health Sci. 2019 Mar;19(1):18011810. [PubMed]

16. International Classification of Sleep Disorders: Diagnostic and Coding Manual. 2nd ed. Westchester, IL. American Academy of Sleep Medicine. 2005. [Internet]

17.van der Meulen MJ, Lobbezoo F, Aartman IH, Naeije M. Self reported oral parafunctions and pain intensity in temporomandibular disorder patients. J Orofac Pain. 2006;20:31-35. [PubMed]

18. Kalaykova S, Lobbezoo F, Naeije M. Effect of chewing upon disc reduction in the temporomandibular joint. J. Orofac. Pain. 2011; 25:49-55. [PubMed]

19. Marpaung C, van Selms MKA, Lobbezoo F. Temporomandibular joint anterior disc displacement with reduction in a young population: Prevalence and risk indicators. Int $J$ Paediatr Dent. 2019 Jan;29(1):66-73. [PubMed]

20. Michelotti A, Cioffi I, Festa P, Scala G, Farella M. Oral parafunctions 
as risk factors for diagnostic TMD subgroups. J Oral Rehabil. 2010; 37(3):157-62. [PubMed]

21.Commisso MS, Martinez-Reina J, Mayo J. A study of the temporomandibular joint during bruxism. Int $J$ Oral Sci. 2014;6(2):116-123. [PubMed]

22.Kijak E, Margielewicz J, Pihut M. Identification of Biomechanical Properties of Temporomandibular Discs. Pain Res Manag. 2020 Oct 7; 2020:6032832. [PubMed]

23. Barrientos E, Pelayo F, Tanaka E,
Lamela-Rey MJ, Fernandez-Canteli A, de Vicente JC. Effects of loading direction in prolonged clenching on stress distribution in the temporomandibular joint. J Mech Behav Biomed Mater. 2020 Dec;112:104029. [PubMed]

24. Mori H, Horiuchi S, Nishimura S, Nikawa H, Murayama T, Ueda K, et al. Three-dimensional finite element analysis of cartilaginous tissues in human temporomandibular joint during prolonged clenching. Arch Oral Biol. 2010 Nov;55(11):879-86. [PubMed]
25.Berger M, Szalewski L, Szkutnik J, Ginszt M, Ginszt A. Different association between specific manifestations of bruxism and temporomandibular disorder pain. Neurol Neurochir Pol. 2017 Jan-Feb; 51(1): 7-11. [PubMed]

26.Garip H, Tufekcioglu S, Kaya E. Changes in the temporomandibular joint disc and temporal and masseter muscles secondary to bruxism in Turkish patients. Saudi Med J. 2018; 39(1):81-85. [PubMed]

Please cite this article as: Kirov D, Doichinova L, Deliverska E, Kirilova J. Assessment of the relationship between temporomandibular joint sounds and bruxism. J of IMAB. 2022 Jan-Mar;28(1):4242-4246.

DOI: https://doi.org/10.5272/jimab.2022281.4246

Address for correspondence:

Dimitar Kirov, Associate Professor, DDS, PhD

Department of Prosthetic Dentistry, Faculty of Dental Medicine, Medical University - Sofia,

1, St. Georgi Sofyiski Str., 1431 Sofia, Bulgaria.

e-mail: dimiterkirov@gmail.com 